\title{
Megavolt discharges triggered and guided with laser filaments
}

\author{
J. Yu, J. Kasparian, E. Salmon and J.-P. Wolf \\ Teramobile Project, LASIM, UMR CNRS 5579,Université Claude Bernard Lyon 1, 69622 Villeurbanne Cedex, France \\ telephone:+334724326 63, fax: +33472445871 and e-mail: jinyu@lasim.univ-lyon1.fr \\ M. Rodriguez and R. Sauerbrey \\ Teramobile Project, Institut für Optik und Quantenelektronik, FSU Jena, Max-Wien-Platz 1, D-07743 Jena, Germany
}

H. Wille and L. Wöste

Teramobile Project, Institut für Experimentalphysik, FU Berlin, Arnimallee 14, D-14195 Berlin, Germany

T. Fujii

Electrical Physics Department, CRIEPI, 2-11-1 Iwado Kita, Komae-shi, Tokyo 201-8511, Japan

Y.-B. André and A. Mysyrowicz

Teramobile Project, Laboratoire d'Optique Appliquée,UMR CNRS 7639, ENSTA-Ecole Polytechnique, 91761 Palaiseau Cedex, France

\section{Klingbeil, K. Rethmeier and W. Kalkner}

Institut für Elektrische Energietechnik, TU Berlin, Einsteinufer 11, D-10587 Berlin, Germany

\begin{abstract}
Long ionized channels appear as a result of nonlinear propagation of femtosecond terawatt pulses. We demonstrate the ability of such filament bundles to trigger and guide megavolt discharges across a gap up to $3.8 \mathrm{~m}$.

$\square 2002$ Optical Society of America

OCIS codes: (010.1300) Atmospheric propagation, (190.7110) Ultrafast nonlinear optics, (350.5400) Plasmas
\end{abstract}

Filaments are self-guiding structures due to the dynamic balance between Kerr self-focusing and plasma-induced defocusing. The Teramobile project, supported by the French CNRS and the German DFG has been launched in 1999 to study the filamentation propagation and to develop applications in the atmosphere, by using this worldwide unique mobile multi-terawatt laser [1]. One of the main applications is lightning control. In this respect, we investigated the triggering and guiding of high voltage discharges, which is the first step towards atmospheric scale experiments. Unlike previous experiments, where discharges were triggered by a plasma focus, we used for the first time long conducting filaments. This opens new perspectives in lightning control. Triggering and guiding have been observed over a gap up to $3.8 \mathrm{~m}$ in air [2]. We show that aiming the laser beam in the vicinity of the two electrodes allows to ohmically bridge the electrodes, which results in (i) a reduced breakdown threshold to $68 \%$ of the value for natural discharges, (ii) a straight guided discharge path, and (iii) an increasing streamer propagation velocity of a factor 3 (Fig.1). In order to extend the plasma lifetime, we are investigating the use of a multi-pulse scheme [3] to reduce electron loss and extend the guided length. Connected to this problematic is triggering discharges in the leader mode [4], which better simulates actual lightning processes.
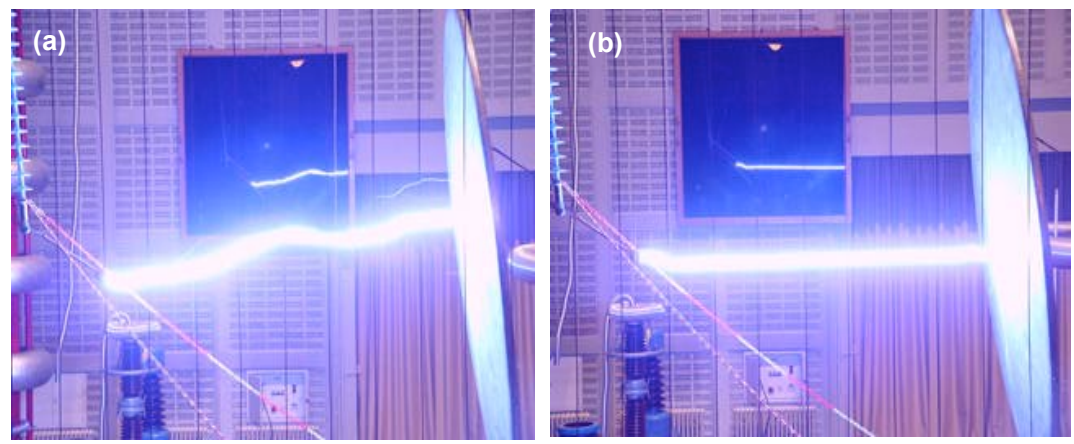

Fig. 1. (a) unguided discharge, (b) guided discharge. 


\section{References}

1. H. Wille, M. Rodriguez, J. Kasparian, D. Mondelain, J. Yu, A. Mysyrowicz, R. Sauerbrey, J.-P. Wolf and L. Wöste, "Teramobile: a mobile femtosecond-terawatt laser and detection system", To appear in European Physical Journal -Applied Physics (2002). See also the Teramobile project web site: www.teramobile.org.

2. M. Rodriguez, R. Sauerbrey, H. Wille, L. Wöste, T. Fujii, Y.-B. André, A. Mysyrowicz, L. Klingbeil, K. Rethmeier, W. Kalkner, J. Kasparian, E. Salmon, J. Yu, J.-P. Wolf, "Triggering and Guiding Megavolt Discharges Using Laser-Induced Ionized Filaments”, Opt. Lett. 27, $772-774$ (2002).

3. J.C. Diels, Xin M. Zhao, "Discharge of lightning with ultrashort laser pulses", United States Patent, number 05175664 (1992).

4. H. Pépin, D. Comptois, F. Vidal, C. Y. Chien, A. Desparois, T. W. Johnston, J. C. Kieffer, B. L. Fontaine, F. Martin, F. A. M. Rizk, C. Potvin, P. Couture, H. P. Mercure, A. Bondiou-Clergerie, P. Lalande, I. Gallimberti, "Triggering and guiding high-voltage large-scale leader discharge with sub-joule ultrashort pulses”, Phys. Plasmas 8, 2532-2539 (2001). 